\title{
Direct instillation of Lactobacillus plantarum into rat bladder inhibits urinary tract infection (UTI) and protects the integrity of urinary organs
}

\author{
Ifeoma M. Ezeonu ${ }^{1 \star}$, Goodness O. Louis ${ }^{1}$, Chinedu A. Eze ${ }^{2}$, Kennedy F. Chah ${ }^{3}$ and Nnnenna \\ E. Ugwu' ${ }^{2}$
}

${ }^{1}$ Department of Microbiology, University of Nigeria, Nsukka, Enugu State, Nigeria.
2Department of Veterinary Surgery, University of Nigeria, Nsukka, Enugu State, Nigeria.
${ }^{3}$ Department of Veterinary Microbiology and Pathology, University of Nigeria, Nsukka, Enugu State, Nigeria.

Accepted 8 January, 2020

\begin{abstract}
Urinary tract infection (UTI) remains one of the commonest clinical problems presenting to medical practitioners. UTIs are frequently treated with antibiotics, which has exacerbated the emergence of resistant strains among uropathogens. Consequently, there is increasing interest in the development of safe alternatives to antibiotics. The use of probiotics has been advocated as a plausible alternative to antibiotics, but questions remain as to their mode of application. This study was therefore conducted to evaluate the ability of Lactobacillus (L.) plantarum, inoculated intraurethrally into rats, to inhibit the establishment of UTI by selected multidrug-resistant uropathogens [E. coli and Klebsiella (K.) pneumonia]. Nine-week old, male, albino rats, were either infected and then treated with L. plantarum or inoculated with L. plantarum before infection. UTI was monitored by determination of bacterial counts from the rats' urine over a period of 14 days, direct culture from- and histological examination of sections of bladder and kidneys of the animals after sacrifice. In untreated rats, both E. coli and K. pneumonia established UTI in the rats within 4 days, with bacterial counts, in urine, increasing from $10^{4} \mathrm{CFU} / \mathrm{ml}$ to about $10^{6} \mathrm{CFU} / \mathrm{ml}$, over the course of 14 days. The infections were accompanied by inflammatory changes in both the bladder and kidneys and bacteria were recovered from sections of the kidneys after sacrifice. In animals infected and then treated with $L$. plantarum, the numbers of the pathogens in urine decreased significantly $(p<0.005)$ to less than $10^{3} \mathrm{CFU} / \mathrm{ml}$, while pre-colonization of the bladder with $L$. plantarum before infection resulted in complete inhibition of the uropathogens. No pathological changes were observed in the urinary organs of the treated rats. These results therefore show that $L$. plantarum can colonize the bladder safely and serve as an effective means of control of UTI.
\end{abstract}

Keywords: UTI, Lactobacillus plantarum, uropathogens, bladder, probiotics, E. coli.

*Corresponding author. Email: ifeoma.ezeonu@unn.edu.ng. Tel: +2348037954649.

\section{INTRODUCTION}

Urinary tract infection (UTI) is defined as the inflammation of the urinary system, which includes the urethra, bladder and kidneys (Manzoor et al., 2016). It remains one of the commonest clinical problems presenting to medical practitioners and recurrent infections in susceptible patients, presents an especially serious challenge
(Hopkins et al., 1995; Naderi et al., 2014; Manzoor et al., 2016). Although both genders are susceptible to the infection, women are mostly vulnerable due to their anatomy and reproductive physiology (Vasudevan, 2014). The commonest pathogens associated with UTI include Escherichia coli, Klebsiella pneumonia and 
Staphylococcus, particularly S. saprophyticus (Lo et al., 2016). It is believed that UTI develops when these bacteria from the intestinal flora sequentially colonize the perineum, vagina, urethra and bladder, and sometimes the kidneys as well, through vesicoureteral reflux (Hopkins et al., 1995; Nosseir et al., 2012). UTIs are frequently treated with antibiotics, which results in an intensive selective pressure over the bacterial populations. This has exacerbated the emergence of resistant strains among uropathogens. Consequently, there is increasing interest in the development of safe alternatives to antibiotics.

The use of probiotics has been advocated as an alternative to antibiotics, under the concept of bacterial interference. The concept of bacterial interference, both passive and active, embodies the use of bacteria of low virulence to compete with and protect against colonization and infection by disease-causing organisms. In addition to producing antimicrobial toxins, probiotics have the ability to adhere to cells, reducing pathogenic bacteria adherence, hence causing pathogen exclusion. Probiotics also interact with epithelial cells and dendritic cells and produce immunomodulatory effects (Sharma et al., 2014).

The application of probiotics and other non-pathogenic bacteria for management of UTI and other infections has been attempted by several groups (Reid et al., 1987; Hatakka and Saxelin, 2008; Deshpande et al., 2011; Darouiche et al., 2011; Darouiche and Hull, 2012), but not taken seriously enough, due to the varying degrees of success recorded and some reported adverse side effects. However, given the persistent public health burden of UTI and the rapid spread of antibioticresistance, the option of probiotics must essentially be given more serious attention. Lactobacillus strains are among the most commonly used microorganisms applied as probiotics for the treatment of infectious diseases in humans (Naderi et al., 2014). Lactobacilli are regarded as generally safe, although rare cases of infection have been reported in immunocompromised individuals (Salminen et al., 1998; Rautio et al., 1999). Lactobacillus strains are known to be predominant colonizers of the adult vagina, where they play a protective role in inhibiting pathogenic bacteria (Kaewsrichan et al., 2006; Ronnqvist, 2007; Akgül and Karakan, 2018). This attribute makes them candidate probiotics to inhibit UTIcausing organisms in the bladder. This study was therefore undertaken to evaluate the ability of Lactobacillus (L.) plantarum to inhibit the establishment of UTI in an in vivo model, using experimental rats challenged with selected multidrug-resistant uropathogens ( $E$. coli and $K$. pneumonia). The specific objectives of the study were: to evaluate the ability of $L$. plantarum inoculated intraurethrally into rats to successfully colonize the bladder; to evaluate the ability of L. plantarum to clear an established UTI; and to evaluate the potential of pre-colonization of the bladder with $L$. plantarum to inhibit establishment of UTI by uropathogens.

\section{MATERIALS AND METHODS}

\section{Isolation of organisms}

Lactobacillus was isolated from High Vaginal Swab (HVS) specimens. HVS specimens were collected from apparently healthy female individuals and inoculated onto De Man-Rogosa- Sharpe (MRS) agar plates. The plates were incubated at $37^{\circ} \mathrm{C}$ for $48 \mathrm{~h}$ under anaerobic conditions with $5 \% \mathrm{CO}_{2}$. Isolated colonies were then picked from each plate and subcultured to obtain pure cultures. The pure cultures were maintained in MRS broth at $4^{\circ} \mathrm{C}$ according to the method described by Manzoor et al. (2016).

Cultures of multidrug-resistant E. coli and Klebsiella pneumonia were obtained from Classic Biomedical Laboratory, Nsukka, and recharacterized.

\section{Morphological and biochemical characterization of isolates}

Suspected Lactobacillus isolates were characterized by Gram staining, catalase test, oxidase test, fermentation of sugars (glucose, xylose, rhamnose, maltose, arabinose, mannose and lactose), growth at different $\mathrm{pH}$ values $(2,3,4,8$ and 9), and tolerance to different sodium chloride $(\mathrm{NaCl})$ concentrations $(2,3,4$, 5 and 6.5\%) as described by Aslam and Qazi (2010).

The E. coli and K. pneumoniae isolates were screened by Gram staining, lactose fermentation, motility testing, citrate utilization testing and methyl red testing according to the methods described in Manual of Microbiology by Kanika (2011).

\section{Genetic identification of Lactobacillus isolates}

Suspected Lactobacillus strains were plated on Nutrient Agar (Oxoid, UK) for $24 \mathrm{~h}$ then subcultured into Nutrient Broth and incubated at $37^{\circ} \mathrm{C}$ for $48 \mathrm{~h}$ under anaerobic conditions with $5 \%$ $\mathrm{CO}_{2}$. Bacterial genomic DNA was extracted and purified using ZR fungal/bacterial DNA miniprep kit (Zymo Research, USA), according to the manufacturer's protocol. The purified DNA was subsequently used for sequencing at Inqaba Biotechnical Company, South Africa. The 16S rDNA amplified PCR product (10 $\mathrm{gg}$ concentration) was used for the sequencing.

\section{Experimental design and inoculation of animals}

A total of forty-eight (48), nine-week old, male albino rats were used. The rats were housed in cages kept in the Faculty of Biological Sciences Animal House and acclimatized for two weeks. Thereafter, the rats were divided into eight (8) groups $(A$ to $H)$ of six rats each. The inoculation and treatment of the groups were as follows: Group A, No inoculation (Control); Group B, intraurethral instillation of $E$. coli only; Group $C$, intraurethral instillation of $L$. plantarum only; Group D, intraurethral instillation of E. coli followed $72 \mathrm{~h}$ later by instillation of L. plantarum; Group E, intraurethral instillation of $L$. plantarum followed $72 \mathrm{~h}$ later by instillation of $E$. coli; Group F, instillation of $K$. pneumonia followed $72 \mathrm{~h}$ later by instillation of L. plantarum; Group G, instillation of $K$. pneumonia alone; and Group $\mathrm{H}$, instillation of $L$. plantarum followed $72 \mathrm{~h}$ later by instillation of $K$. pneumonia. Inoculation of the animals was carried out with the assistance of a veterinary surgeon from the Faculty of Veterinary Medicine, University of Nigeria, Nsukka, following the accepted guidelines for handling of animals. The rats were anesthetized by inhalation of chloroform for 1 minute. After sterilization of the periurethral area with $70 \%$ ethanol, a sterile 24 - 
gauge cannula catheter was inserted into the bladder through the urethra. A $50 \mu$ inoculum containing about $1 \times 10^{4}$ cells suspended in Phosphate Buffered Saline (PBS) was then infused into the bladder through the catheter, over a period of $30 \mathrm{~s}$, with a syringe (Asahara et al., 2001). The rats were monitored over a period of 14 days.

\section{Determination of bacterial counts $(\mathrm{CFU} / \mathrm{ml})$ in urine}

Urine samples from experimental rats were collected on Days 4 and 11 of the monitoring period. To collect the urine sample from each animal, the anus of the rat was clogged with cotton wool and sealed with tape, after which the entire periurethral area, tail and legs were swabbed with $70 \%$ ethanol. The rat was administered $0.5 \mathrm{ml}$ of furosemide (for stimulation of urine production) intramuscularly and placed back in a cage with an opening at the bottom and a collection plate placed beneath the opening. The urine sample was then collected after 10 to $15 \mathrm{~min}$ using a sterile syringe. Enumeration of the uropathogens ( $E$. coli and $K$. pneumoniae) and $L$. plantarum from the urine was on Cystine Lactose Electrolyte Deficient (CLED) agar and MRS agar, respectively. The urine samples were serially diluted and plated on the agar media. The MRS plates were incubated anaerobically at $37^{\circ} \mathrm{C}$ for $24 \mathrm{~h}$, while the CLED plates were incubated aerobically at $37^{\circ} \mathrm{C}$ for $24 \mathrm{~h}$ to determine the bacterial colony counts (Liu et al., 2016).

\section{Organ culture}

To determine the number of viable bacteria in urinary organs, the rats in each group were sacrificed on Day 14 after being anesthetized with chloroform. Bladders and kidneys were removed aseptically, cut longitudinally and swabbed with sterile cotton swabs. The swabs were cultured onto CLED agar and MRS agar media and then incubated as previously described (Asahara et al., 2001).

\section{Histopathological examination of organs}

This was carried out as described by Bancroft and Gamble (2008). On Day 14 of the experiment, kidney and bladder samples from three rats in each group were collected and fixed in $10 \%$ neutral buffered formalin to arrest metabolic activity in the tissues, avoid autolysis and protein precipitation thus preventing enzymatic digestion of dead tissues. The fixed tissues were passed through several changes of alcohol; $70 \%$ alcohol for $24 \mathrm{~h}, 90 \%$ alcohol for $12 \mathrm{~h}$ and through absolute alcohol to remove water from the fixed tissues. Thereafter, there was complete infiltration of tissues by paraffin. The tissues were then passed through xylene for $3 \mathrm{~h}$ to prevent shrinkage and tissue brittleness in paraffin. After tissue processing, tissues were embedded or blocked out using the Leukhand embedded mould. The $L$ pieces were arranged on an aluminum base to form a rectangle. The molten paraffin was then poured into the moulds and the selected surfaces of the tissues embedded with the aid of a pair of blunt end forceps and allowed to set. The embedded tissues were separated into different blocks and then attached to wooden blocks with the aid of an electric spatula. The blocks were then trimmed using a rotary microtome and knife. At the end of each trimming, the blocks were arranged on ice trays in order to cut thin sections using the rotary microtone at a thickness of $5 \mu \mathrm{m}$. Sections were then collected with the help of a camel hair brush and then placed on the slide. Sections were flooded with $20 \%$ alcohol in order to spread out folds on the sections and then floated out on a water bath with a temperature of 5 to $10^{\circ} \mathrm{C}$ below the melting point of the wax used. The sections were picked and floated on a water bath and then picked with a pre- labeled slide. The slides were dried on a hot plate at a temperature of 5 to $10^{\circ} \mathrm{C}$ degree centigrade above the melting point of wax used. These were left on the hot plate for $15 \mathrm{~min}$. Thereafter, the sections were stained with haematoxylin and eosin and examined using a Moticam Images Plus 2.0 digital camera (Motic China Group Ltd. 1999-2004).

\section{Statistical analysis}

Data on bacterial numbers in urine and organ sections were expressed as mean \pm standard deviation (SD). The data were analyzed for significant differences using one-way Analysis of Variance (ANOVA) at 95\% confidence level (Dashe et al., 2013).

\section{RESULTS}

\section{Characteristics of Lactobacillus isolates from HVS}

Five isolates were obtained, which had expected features of Lactobacillus. These isolates were Gram positive tiny rods that produced slightly raised, grayish-white colonies of about $0.5 \mathrm{~mm}$ diameter on MRS agar. They were catalase and oxidase negative and fermented sugars to varying extents, without gas production. One isolate was selected for the animal studies, based on its in vitro inhibitory activity against test organisms, as well as wide range of $\mathrm{pH}$ and $\mathrm{NaCl}$ tolerance. The isolate was identified by $16 \mathrm{~S}$ rDNA sequencing as Lactobacillus plantarum subsp plantarum (Acession number CP025991.1).

\section{Growth of L. plantarum in the rat bladder}

In animals intraurethrally inoculated with L. plantarum alone (Group C), colonization was established and sustained for at least 4 days. However, by Day 11, the cells could not be detected (Figure 1).

\section{Growth and interaction of E. coli, K. pneumoniae and L. plantarum in the Urinary Tract of Inoculated Rats}

Animals in group B were inoculated with E. coli on Day 0. By Day 4, infection was established, with cell numbers in urine increasing significantly $(P<0.05)$ by almost two log values by Day 11 (Figure 2: B). In animals infected and later treated with L. plantarum (Group D), infection with $E$. coli was established, but with introduction of $L$. plantarum on Day 4, there was significant $(\mathrm{P}<0.05)$ reduction in the number of $E$. coli cells (Figure 2: D1), while the number of L. plantarum was sustained up to Day 11 (Figure 2: D2). In animals pre-colonized with $L$. plantarum before inoculation with E. coli (Group E), colonization and growth of the E. coli was severely inhibited (Figure 2: E1), while the growth of L. plantarum was sustained (Figure 2: E2).

Animals in group $\mathrm{G}$ were inoculated with $K$. pneumoniae on Day 0. By Day 4, infection was 


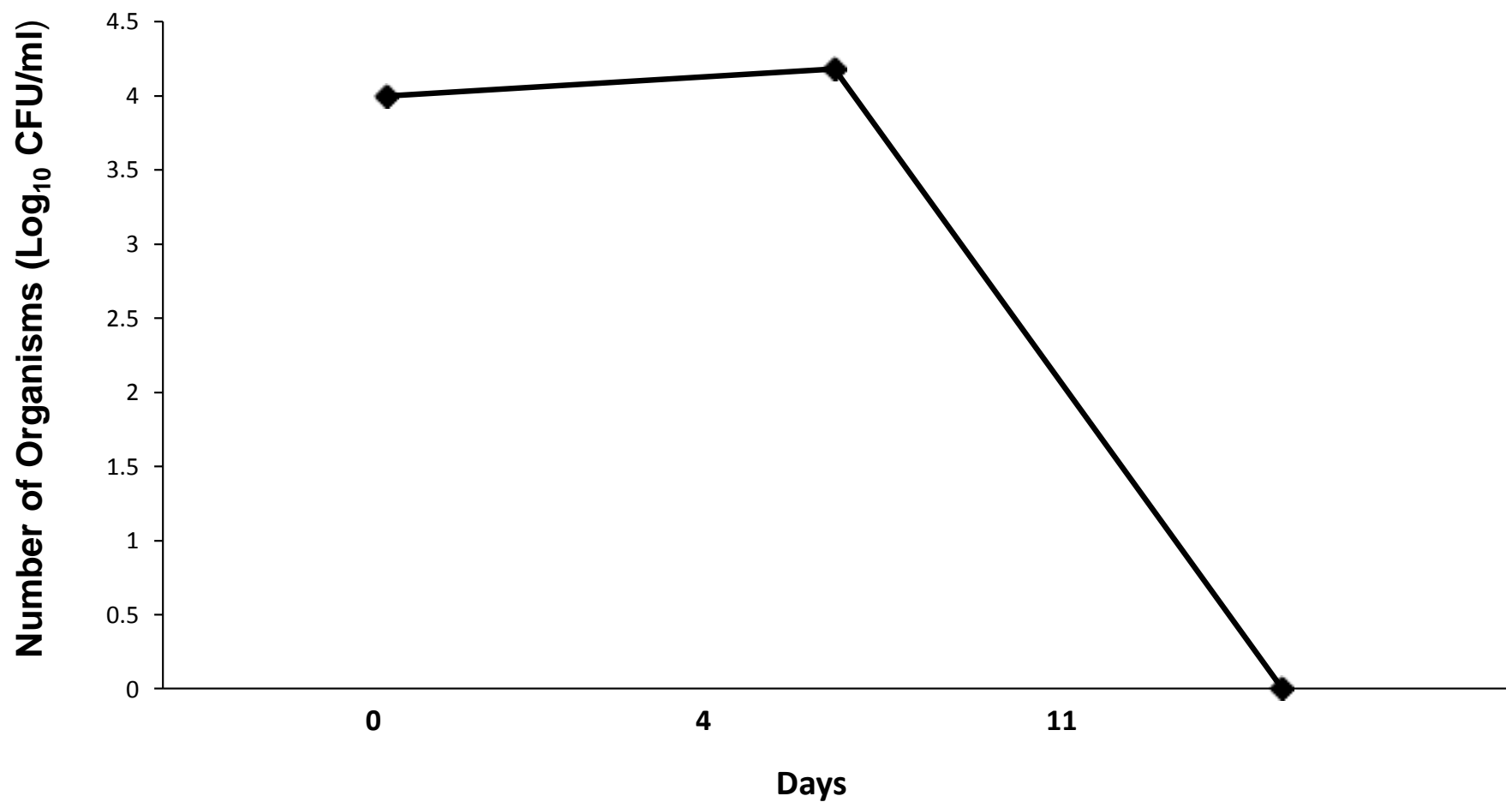

Figure 1. Growth of Lactobacillus plantarum in the rat bladder. Note the sharp drop in number of cells after 4 days until cells were no longer recovered in urine by Day 11.

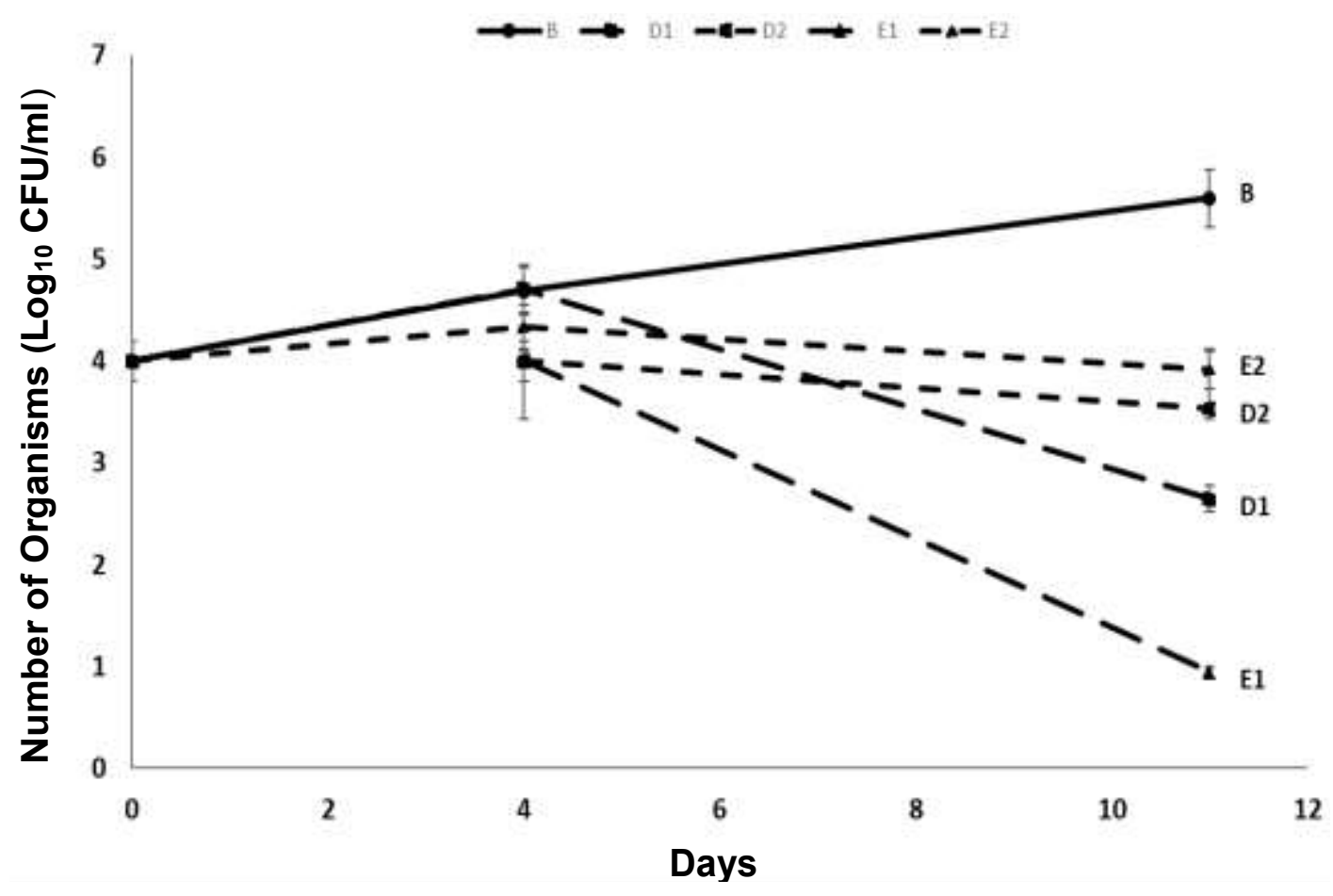

Figure 2. Growth and interaction of E. coli and L. plantarum in the bladder. B, represents counts from urine of group of animals infected by intraurethral inoculation of $E$. coli. D, represents groups in which infection was followed by treatment with L. plantarum (D1, E. coli; D2, L. plantarum). E represents groups in which bladder was pre-colonized with L. plantarum before infection (E1, E. coli; E2, L. plantarum). Note inhibition of $E$. coli with addition of $L$. plantarum (D1) and severe inhibition when bladder was precolonized with L. plantarum (E1). 
established and cell numbers in urine had increased significantly $(\mathrm{P}<0.05)$, before declining slightly again (Figure 4: G) by Day 11. In animals infected with $K$. pneumonia and treated with L. plantarum 72 hours later (Group F), infection was established, but with introduction of $L$. plantarum, there was a sharp and significant $(\mathrm{P}<0.05)$ reduction in the number of $K$. pneumoniae (Figure 3: F1) by more than $2 \log _{10} \mathrm{CFU} / \mathrm{ml}$, while the number of $L$. plantarum was sustained up to Day 11 (Figure 3: F2). In animals pre-colonized with L. plantarum before inoculation with $K$. pneumoniae, (Group H), colonization and growth of the $K$. pneumoniae was severely inhibited (Figure 3: $H 1$ ), while the growth of $L$. plantarum was sustained (Figure 3:H2).

\section{Recovery of bacteria from organ cultures}

The experimental rats were sacrificed and organs cultured after 14 days of observation. In animals infected with $E$. coli $(\mathrm{B})$, the uropathogen was isolated from the bladders of all the animals and kidneys of some animals after sacrifice. K. pneumoniae was also isolated from the bladders of Group $\mathrm{G}$ animals, but not from the kidneys. In Groups D and E as well as Groups $F$ and $H$, where animals were treated or pre-colonized with L. plantarum, the numbers of uropathogens re-isolated from the bladder were significantly reduced. There was also no isolation from the kidneys. Furthermore, there were significantly $(P<0.05)$ more $L$. plantarum cells compared to the number of $E$. coli and $K$. pneumoniae isolated from the bladders of the treated animals (Table 1).

\section{Pathological changes in the bladder and kidneys of experimental rats}

The major histopathological findings were observed in the bladder and kidneys of rats in groups B and G. The lesions in the bladder of both groups were those of inflammatory material and proteinaceous plug in the lumen and submucosa of the bladder (Figure 4), while the lesions in the kidneys were those of pyelonephritis or suppurative nephritis. These included solitary or multifocal discrete microscopic aggregations of neutrophils either intraluminal in tubules or in the interstitium. These were mixed with other inflammatory cells such as plasma cells and macrophages (Figure 5). There was also mild inflammation of the renal pelvis in some animals. The bladders and kidneys in all other groups of rats treated with $L$. plantarum, as well as control rats, were apparently normal.

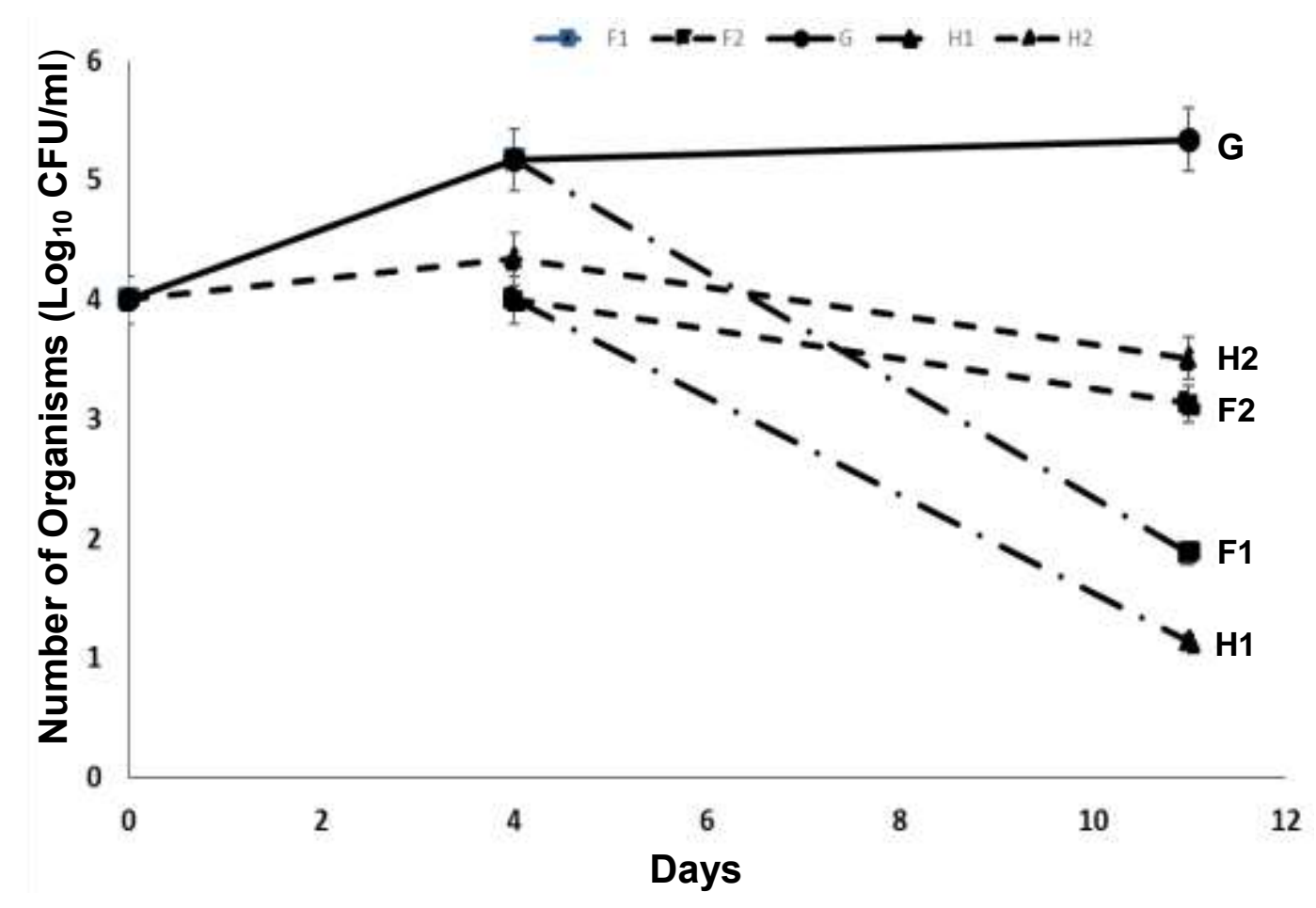

Figure 3. Growth and interaction of $K$. pneumoniae and L. plantarum in the bladder. G represents counts from urine of group of animals infected by intraurethral inoculation of $K$. pneumoniae. $F$ represents group in which infection was followed by treatment with L. plantarum (F1, K. pneumoniae; F2, L. plantarum). H represents group in which bladder was pre-colonized with $L$. plantarum before infection ( $\mathrm{H} 1, K$. pneumoniae; $\mathrm{H} 2$, L. plantarum). Note inhibition of $K$. pneumoniae with addition of $L$. plantarum (F1) and severe inhibition when bladder was pre-colonized with L. plantarum $(\mathrm{H} 1)$. 

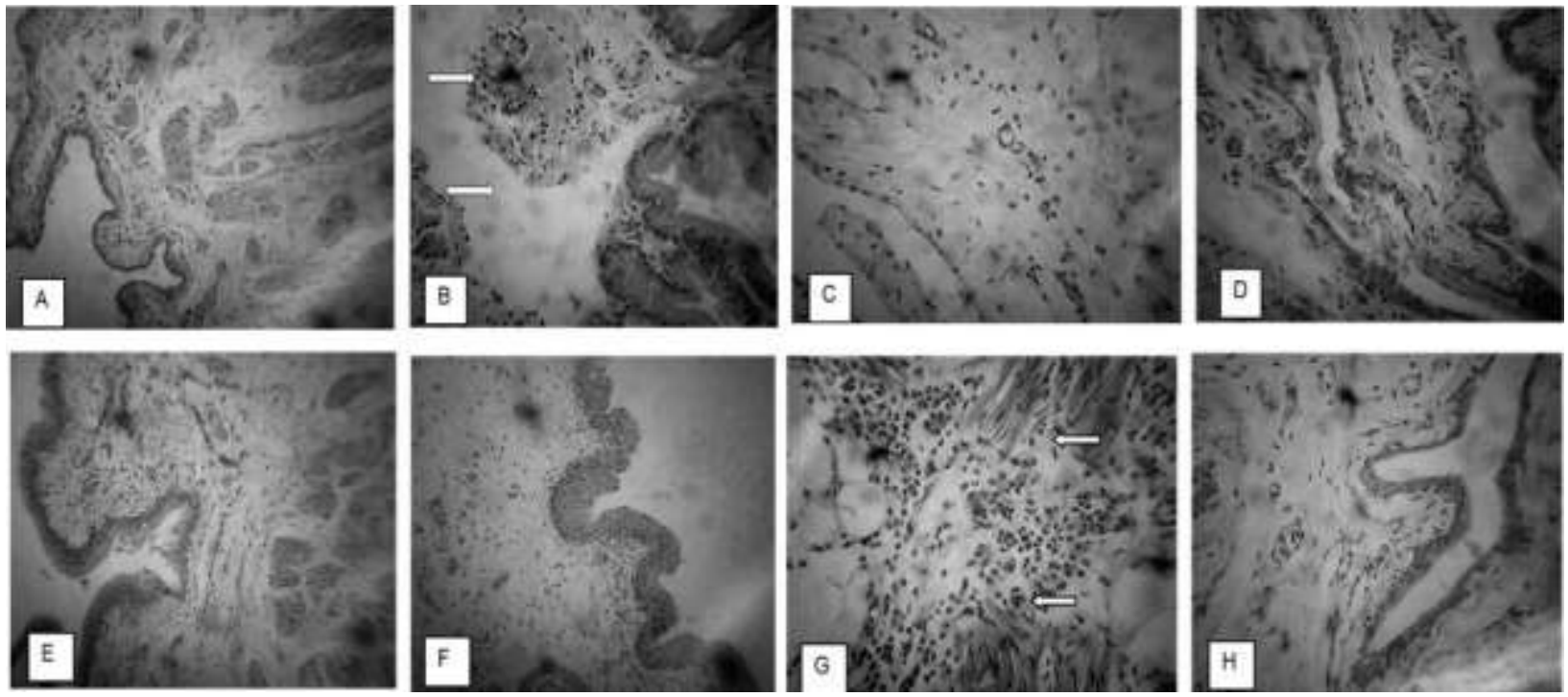

Figure 4. Phptpmicrograph of the bladder from experimental rats in groups $\mathrm{A}$ to $\mathrm{H}$, showing presence of fibrinous exudates mixed with cellular debris and infiltration of plasma cells, macrophages and polymorphonuclear cells (white arrows) within lumen of the bladder in B (animals infected with $E$. coli) and mild inflammatory cells infiltration of the submucosa of the bladder (white arrows) in G (animals infected with K. pneumonia). H and E Stain X400.

Table 1. Re-isolation of microorganisms from the bladders and kidneys of experimental rats.

\begin{tabular}{lllllc}
\hline & \multicolumn{4}{c}{ Number of cells recovered in culture } \\
\cline { 2 - 3 } \cline { 5 - 6 } Group & \multicolumn{2}{c}{ L. plantarum } & & \multicolumn{2}{c}{ Uropathogens } \\
\cline { 2 - 3 } \cline { 5 - 6 } & Bladder & 0.00 & & 0.00 & Kidney \\
\hline A & 0.00 & 0.00 & & $2.1 \times 10^{4} \pm 85.39$ & $5.0 \times 10^{1} \pm 0.00$ \\
B & 0.00 & 0.00 & & 0.00 & 0.00 \\
C & 0.00 & 0.00 & & $3.0 \times 10^{1} \pm 0.00$ & 0.00 \\
D & $4.8 \times 10^{4} \pm 2.4 \times 10^{2}$ & 0.00 & & $2.0 \times 10^{1} \pm 0.00$ & 0.00 \\
E & $4.8 \times 10^{2} \pm 2.3 \times 10^{2}$ & 0.00 & 0.00 \\
F & $4.1 \times 10^{2} \pm 1.6 \times 10^{2}$ & 0.00 & & $2.0 \times 10^{1} \pm 14.1$ & 0.00 \\
G & 0.00 & 0.00 & & $2.7 \times 10^{2} \pm 1.3 \times 10^{2}$ & 0.00 \\
H & $1.9 \times 10^{2} \pm 73.9$ & 0.00 & & $3.0 \times 10^{1} \pm 0.00$ & 0.00 \\
\hline
\end{tabular}

\section{DISCUSSION}

It has been suggested that Lactic Acid Bacteria (LAB) inhibit other bacteria through a variety of mechanisms, including production of antimicrobial compounds such as hydrogen peroxide, lactic acids, acetic acids, and ribosomally-synthesized peptides known as bacteriocins (Reid, 2016). LAB, especially Lactobacillus strains, are known to play protective roles in the urogenital tract (De Gregorio et al., 2014; Reid, 2016; Akgül and Karakan, 2018). They have also been applied as vaginal suppositories in women for the treatment of recurrent UTI (Borchert et al., 2008; Akgül and Karakan, 2018). This study was carried out to evaluate the ability of $L$. plantarum, inoculated intraurethrally into rat bladder, to inhibit the establishment of UTI by selected multidrugresistant E. coli and K. pneumonia.
Lactobacillus isolates from HVS were screened in vitro for inhibitory activity against selected multi-drug resistant uropathogens and the isolates showing activity were identified by $16 \mathrm{~S}$ rDNA sequencing. The isolate exhibiting the highest activity was identified as $L$. plantarum. This finding supports other studies that have reported the inhibitory action of $L$. plantarum against UTI-causing organisms (Lim et al., 2009; Selvamohan and Sujitha, 2010; Poppi et al., 2015; Liu et al., 2016; Oldak et al., 2017).

In this study, the experiments were designed to investigate three potentials: the ability of $L$. plantarum to colonize the bladder without disease; the ability of $L$. plantarum to clear an established UTI; and the ability of L. plantarum to prevent establishment of UTI. The results showed that $L$. plantarum was able to colonize the rats' urinary tracts, when introduced intraurethrally. However, 

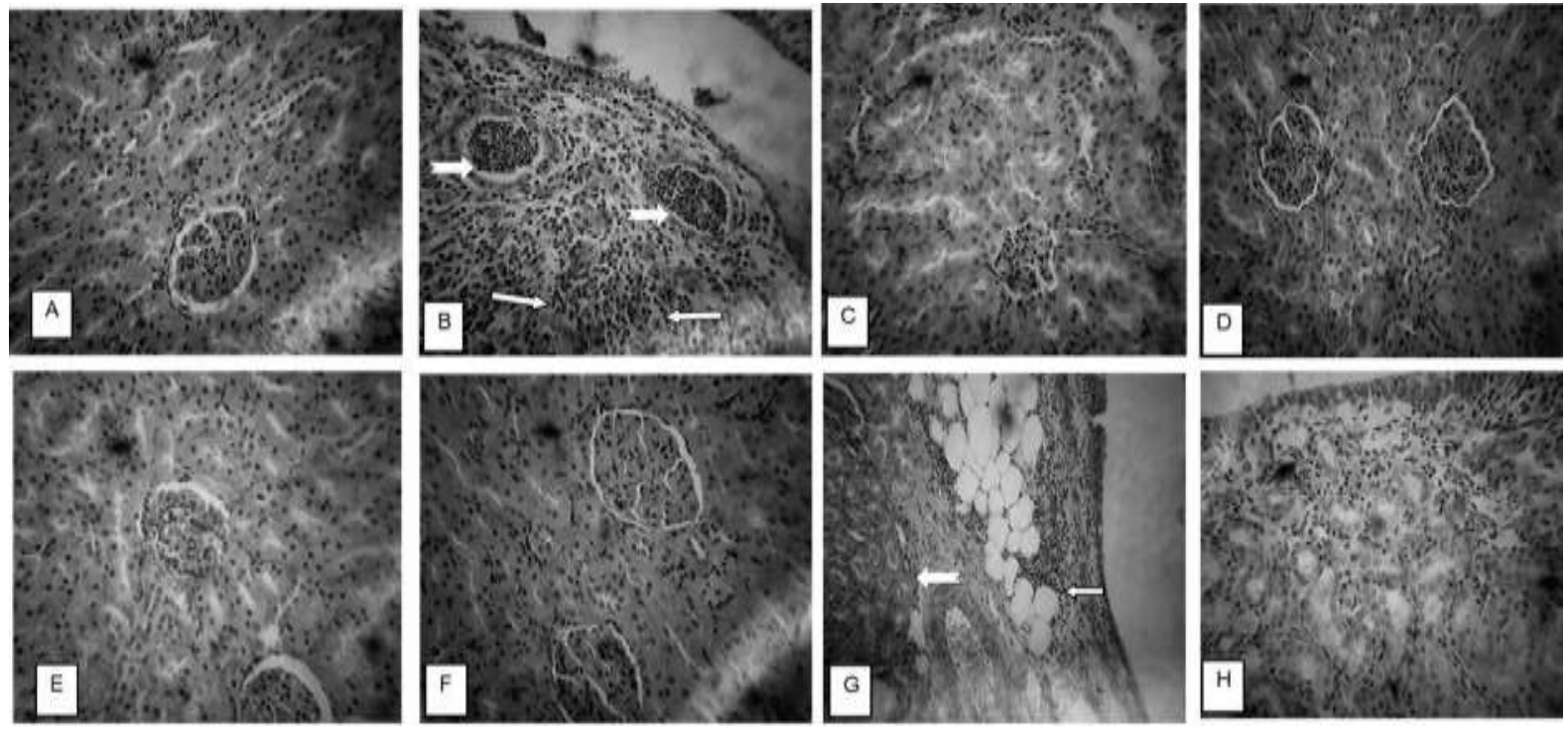

Figure 5. Photomicrograph of the kidney from experimental rats in groups $\mathrm{A}$ to $\mathrm{H}$. Note severe infiltration of plasma cells, macrophages and polymorphonuclear cells (thin arrows) within the medulla and renal pelvis with casts made up of microabscesses (fat arrows) in B (animals infected with E. coli) and G (animals infected with K. pneumoniae) while others are apparently normal. $\mathrm{H}$ and $\mathrm{E}$ Stain $\mathrm{x} 400$.

the colonization was not sustained in the absence of other organisms, as the cell numbers in urine reduced steadily from the fourth day after inoculation, until there was complete clearance by the eleventh day as seen in Figure 1. In contrast, both $E$. coli and K. pneumonia colonized the bladders of the animals and proliferated successfully, with numbers in urine increasing by between 1 and $2 \log _{10} \mathrm{CFU} / \mathrm{ml}$ for both organisms (Figures 2 and 3 ). The infection with $E$. coli was more pronounced and the organism ascended the urinary tract to the kidney, as evidenced by the recovery of organisms from kidney culture. Successful infection of the bladder by intraurethral instillation of the uropathogens, in this study, is in line with the reports of Hopkins et al. (1995), who had previously reported successful colonization of experimental rats within 2 days using this method.

The difficulty in establishing Lactobacillus within the urinary bladder has been reported previously (Reid et al., 1989; Hagberg et al., 1989), and it is said to be one of the reasons why studies on the instillation of probiotics into the bladder for the control of UTI were abandoned (Reid, 2016). Although this difficulty in sustaining colonization by Lactobacillus was considered to be a drawback by these earlier investigators, in this study it is considered to be a positive and important finding, particularly for actual clinical application. In patients for whom probiotic instillation may be indicated, including patients with rUTI, post-operative catheterized patients and patients undergoing long-term catheterization, development of UTI is almost a certain occurrence without intervention. For such cases, therefore, the probiotic may be applied as a preventive measure, to control the development of UTI, but where infection eventually does not occur, the
Lactobacillus may simply wash out with no risk whatsoever of causing harm to the host. This suggestion is further strengthened by histopathological results, which showed that the bladders and kidneys of the rats instilled with Lactobacillus alone were remained normal after 14 days, proving that the presence of $L$. plantarum in the bladder did not serve as a tool for promotion of UTI. It is worthy of note that the growth of $L$. plantarum was sustained only in the presence of the uropathogens. This suggests a dynamic and complex relationship between these organisms, which might require further investigation. It could mean that some substances produced by the uropathogens, such as vitamins, are required for the growth of the lactobacilli and might explain why, even in the female vagina and other environments where lactobacilli inhibit other organisms, it is never a complete elimination.

The therapeutic potential of bladder instillation of $L$. plantarum was evident from the results in Figures 2 and 3. In animals in which UTI was established before introduction of $L$. plantarum, introduction of the probiotic resulted in an almost immediate reduction in numbers of the uropathogens in the bladder as indicated by bacterial counts in urine (Figures 2D1 and 3F1). Histopathological examination of the bladder and kidney tissues of these animals also showed that the tissue architecture was normal in comparison to those of untreated rats which showed evidence of inflammation. In addition to its therapeutic potential, $L$. plantarum could also exhibited the potential to serve as a preventive tool against establishment of infection by the urinary tract pathogens. Instillation of the probiotic into the bladder before challenge with uropathogens resulted in complete 
inhibition of growth of the uropathogens, as the organisms were steadily cleared from the urinary tract (Figures 2E1 and 3H1). The uropathogens were also prevented from ascending the urinary tract or causing inflammation.

The results from this study support the reports from in vitro studies by other investigators, as previously stated, but do not quite agree with the results of de Llano et al. (2017), in whose study L. plantarum was not as active as two other strains against test uropathogenic strains. This may however be attributed to the fact that their strain was isolated from milk unlike the strain in this study, which was isolated from HVS. It is noteworthy that the two other active strains in the de Llano study were HVS isolates.

Previous attempts to control UTI by the application of probiotics have involved the oral administration of probiotic strains with the ability to colonize the intestinal tract and vagina and subsequently reach the urinary tract. The success of this technique however seemed to vary from host to host and depended on the host's avoidance of habits such as use of spermicides, which disrupt the vaginal flora (Marelli et al., 2004). These inconsistencies in the results from various studies have raised questions concerning what properties of the lactobacilli are actually important for activity, optimal dosage, duration of application and even mode of delivery (Marelli et al., 2004; Mokoena, 2017). It has even been suggested that new methods of application should be investigated (Mokoena, 2017). The present study is perhaps the first in recent times to attempt delivery of the probiotics by direct instillation into the bladder and the results are quite encouraging for clinical application. It is also worthy of note that the $L$. plantarum was effective against multidrug resistant uropathogens, which is a promising development for the fight against multidrug resistance.

\section{CONCLUSION}

The results in this study provide powerful support for both therapeutic and prophylactic application of intraurethral instillation of $L$. plantarum in the management of UTIs, particularly in cases of diabetic patients, women with rUTI, post-operative catheterization and long-term catheterization where urgent intervention is often necessary. The study further shows that the source of the probiotic and mode of application may be important factors in the application of probiotics for treatment. It is recommended that this application be carried on to clinical trials to ascertain once and for all the usefulness of probiotics in this manner.

\section{REFERENCES}

Akgül T, Karakan T, 2018. The role of probiotics in women with recurrent urinary tract infections. Turk J Urol, 44: 377-83.

Asahara T, Nomoto K, Watanuki M, Yokokura T, 2001. Antimicrobial activity of intraurethrally administered probiotic Lactobacillus casei in a murine model of Escherichia coli urinary tract infection. Antimicrob Agents Chemother, 45: 1751-1760.

Aslam S, Qazi JI, 2010. Isolation of acidophilic lactic acid bacteria antagonistic to microbial contaminants. Pakistan J Zool, 42: 567-573.

Bancroft JD, Gamble M, 2008. Theory and practice of histological techniques. $6^{\text {th }}$ Edition. Churchill Livingstone, Philadelphia.

Borchert D, Sherida L, Papatsoris A, Faruquz Z, Barua JM, Junaid I, Pati Y, Chinegwundoh F, Buchholz N, 2008. Prevention and treatment of urinary tract infection with probiotics: review and research perspective. Indian J Urol, 24: 139-144.

Darouiche RO, Green BG, Donovan WH, Chen D, Schwartz M, Merritt J, Mendez M, Hull RA, 2011. Multicenter randomized controlled trial of bacterial interference for prevention of urinary tract infection in patients with neurogenic bladder. Urology, 78: 341-346.

Darouiche RO, Hull RA, 2012. Bacterial interference for prevention of urinary tract infection. Clin Infect Dis, 55: 1400-1407.

De Gregorio PR, María S, María CL, María N, 2014. In vitro and in vivo effects of beneficial vaginal lactobacilli on pathogens responsible for urogenital tract infections. J Med Microbiol, 63: 685-696.

de Llano DG, Arroyo A, Cárdenas N, Rodríguez JM, Moreno-Arribas MV, Bartolomé B, 2017. Strain-specific inhibition of the adherence of uropathogenic bacteria to bladder cells by probiotic Lactobacillus spp., Pathogen Dis, 75(4): $\mathrm{ftx} 043$.

Deshpande G, Rao S, Patole S, 2011. Progress in the field of probiotics: year 2011. Curr Opin Gastroenterol, 27:13-18.

Hagberg L, Bruce AW, Reid G, Svanborg Eden C, Lincoln K, LidinJanson G, 1989. Colonization of the urinary tract with live bacteria from the normal fecal and urethral flora in patients with recurrent symptomatic urinary tract infections. In: Kass EH, Svanborg Eden C, editors. Host- parasite interactions in urinary tract infections. Chicago: University of Chicago Press, pp. 194-197.

Hatakka K, Saxelin M, 2008. Probiotics in intestinal and non-intestinal diseases. Curr Pharmacol Design, 14:1351-1367.

Hopkins WJ, Hall JA, Conway BP, Uehling DT, 1995. Induction of urinary tract infection by intraurethral inoculation with Escherichia coli: refining the murine model. J Infect Dis, 171: 462-465.

Kaewsrichan J, Peeyananjarassri K, Kongprasertkit J, 2006. Selection and identification of anaerobic lactobacilli producing inhibitory compounds against vaginal pathogens. FEMS Immunol Med Microbiol, 48: 75-83.

Kanika S, 2011. Manual of Microbiology, 2nd ed. Ane Books Pvt, Ltd, Darya Ganj, New Delhi, India, pp. 183-184.

Lim IS, Lee HS, Kim WY, 2009. The effect of lactic acid bacteria isolates on the urinary tract pathogens to infants in vitro. J Korean Med Sci, 24: 57-62.

Liu $\mathrm{Y}$, Cheng-Ying $\mathrm{H}$, Chun-Chin $\mathrm{H}$, Cheng-Chih $\mathrm{T}$, 2016. Inhibitory effect of lactic acid bacteria on uropathogenic Escherichia coliinduced urinary tract infections. J Probiotics Health, 4: 2.

Lo J, Choi WH, Chan JYH, Lange D, 2016. Overview of urinary tract infections. In: The Role of Bacteria in Urology, Lange D and Chew B (Eds). Springer International Publishing Switzerland, pp 7-31.

Manzoor AM, UI-Haq I, Baig S, Qazi JI, Seratlic S, 2016. Efficacy of locally isolated lactic acid bacteria against antibiotic-resistant uropathogens. Jundishapur J Microbiol, 9: e18952.

Marelli G, Papleo E, Ferrari A, 2004. Lactobacilli for prevention of urogenital infections: a review. Eur Rev Med Pharmacol Sci, 8: 87-95.

Mokoena MP, 2017. Lactic acid bacteria and their bacteriocins: classification, biosynthesis and applications against uropathogens: a mini-review. Molecules 22(8): 1255.

Naderi A, Kasra-Kermanshahi R, Gharavi S, Fooladi AAI, Alitappeh MA, Saffarian P, 2014. Study of antagonistic effects of Lactobacillus strains as probiotics on multi drug resistant (MDR) bacteria isolated from urinary tract infections (UTIS). Iran J Basic Med Sci, 17: 201208.

Nosseir SB, Lind LR, Winkler HA, 2012. Recurrent uncomplicated urinary tract infections in women: a review. J Womens Health, 21: 347-354.

Oldak A, Dorota Z, Anna R, Danuta K, 2017. Comparison of antibacterial activity of Lactobacillus plantarum strains isolated from two different kinds of regional cheeses from Poland. Biomed Res Int, 17: 1-10.

Poppi LB, Rivaldi JD, Coutinho TS, Astolfi-Ferreir CS, Antonio J, 
Ferreira P, Mancilha IM, 2015. Effect of Lactobacillus sp. isolates supernatant on Escherichia coli O157:H7 enhances the role of organic acids production as a factor for pathogen control. Pesq Veterinary of Brasil, 35: 353-359.

Rautio M, Jousimies-Somer H, Kauma H, Pietarinen I, Saxelin M, Tynkkynen S, Koskela M, 1999. Liver abscess due to a Lactobacillus rhamnosus strain indistinguishable from L. rhamnosus strain GG. Clin Infect Dis 28: 1159-1160.

Reid G, 2016. Bacteria in the genitourinary tract: the microbiota and probiotics. In: The Role of Bacteria in Urology, Lange D and Chew B (Eds). Springer International Publishing Switzerland, pp 1-6.

Reid G, Cook RL, Bruce AW, 1987. Examination of strains of lactobacilli for properties which may influence bacterial interference in the urinary tract. J Urol, 138: 330-335.

Reid G, Cook RL, Hagberg L, Bruce AW, 1989. Lactobacilli as competitive colonizers of the urinary tract. In: Kass $\mathrm{EH}$, Svanborg Eden C, editors. Host- parasite interactions in urinary tract infections. Chicago: University of Chicago Press, pp. 390-396.

Salminen S, Ouwehand AC, Isolauri E, 1998. Clinical application of probiotic bacteria. Int Dairy J, 8: 563-572.

Selvamohan T, Sujitha $S, 2010$. Antimicrobial activity of a probiotic Lactobacillus plantarum against urinary tract infection (UTI) causing pathogens. Der Pharmacia Lettre, 2: 432-440.

Sharma J, Chauhan DS, Goyal A, 2014. Enhancement of antimicrobial activity of antibiotics by probiotics against Escherichia coli - an in vitro study. Adv Appl Sci Res, 5:14-18.

Vasudevan R, 2014. Urinary tract infection: An overview of the infection and the associated risk factors. J Microbiol Exper, 1: 0008.
Citation: Ezeonu IM, Louis GO, Eze CA, Chah KF, Ugwu NE, 2020. Direct instillation of Lactobacillus plantarum into rat bladder inhibits urinary tract infection (UTI) and protects the integrity of urinary organs. Microbiol Res Int, 8(1): 1-9. 